\title{
One-dimensional photonic crystal waveguide based on the SOI platform for transverse magnetic polarization-maintaining devices
}

\author{
Nikolay Lvovich Kazanskiy ${ }^{1,2}$, Muhammad Ali Butt, ${ }^{* 1,3}$ \\ ${ }^{1}$ Department of Technical Cybernetics, Samara National Research University, 34 Moskovkoye Shosse, Samara \\ 443086, Russia \\ ${ }^{2}$ Institute of RAS-Branch of the FSRC "Crystallography and Photonics" RAS, 151 Molodogvardeiskaya, Samara \\ 443001, Russia \\ ${ }^{3}$ Institute of Microelectronics and Optoelectronics, Warsaw University of Technology, Koszykowa 75, 00-662 \\ Warszawa, Poland
}

Received August 26, 2020; accepted September 23, 2020; published September 30, 2020

\begin{abstract}
In this letter, a TM-polarization C-band pass of onedimensional photonic crystal strip waveguide (1D-PCSW) is presented. The waveguide structure is based on a silicon-on-insulator platform which is easy to realize using standard CMOS technology. A numerical study is conducted via the 3D-finite element method (FEM). The transmittance and polarization extinction ratio $(P E R)$ is enhanced by optimizing the geometric parameters of the device. As a result, a TM polarized light can travel in the waveguide with $\sim 2 \mathrm{~dB}$ loss for all the $\mathrm{C}$ band telecommunication wavelength window whereas the TE polarized light suffers a high transmission loss of $>30 \mathrm{~dB}$. As a result, a PER of $\sim 28.5 \mathrm{~dB}$ can be obtained for the whole C-band wavelengths range. The total length of the proposed device is around $8.4 \mu \mathrm{m}$ long including a 1 $\mu \mathrm{m}$ silicon strip waveguide segment on both ends. Based on our study presented in this paper, several photonic devices can be realized where strict polarization filtering is required.
\end{abstract}

Several integrated photonics devices that work on transverse-magnetic (TM) polarized light has an efficient performance [1]. Besides, TM polarized light is practical for sensing applications, as its evanescent field penetrates deeper to the top and bottom cladding compared to that of the transverse electric (TE) polarized light. Furthermore, devices used for polarization multiplexing require TM polarized light to facilitate most of the channel capacity [2]. That is why we can say that TM polarization pass devices are essential for designing proficient Photonic Integrated Circuits (PICs). Currently, most of the PICs are designed based on a silicon-on-insulator (SOI) platform to take advantage of high index contrast of silicon $(\sim n=3.48$ at $1550 \mathrm{~nm})$ to silicon dioxide $(\sim \mathrm{n}=1.44$ at $1550 \mathrm{~nm})$, high structural birefringence, significant optical nonlinearities, and, its well-matured CMOS fabrication technologies [35].

Recently, several high performance SOI-based TM polarization filters have been proposed. For instance: a $10 \mu \mathrm{m}$ long silicon strip waveguide has been optimized to produce a TM polarization filter with a polarization extinction ratio $(P E R)$ of $26 \mathrm{~dB}$ [6]. A TM pass

*E-mail: ali_ciit_engineer@yahoo.com polarization filter (length $=26 \mu \mathrm{m})$ has been proposed, capable of providing $20 \mathrm{~dB}$ PER [7]. The TM polarization pass device based on a Hybrid plasmonic waveguide provides a $P E R$ of $20 \mathrm{~dB}$ in $\sim 7.5 \mu \mathrm{m}$ length waveguide [8]. A $27 \mathrm{~dB} P E R$ of TM to the TE polarized light has been achieved in a propagation length of $9 \mu \mathrm{m}$ by using a sub-wavelength grating-based structure [9]. Moreover, a multilayer TM polarizer based on aluminium-doped zinc oxide has been proposed, which gives a PER of $22 \mathrm{~dB}$ [10]. However, these are undeniably high performance polarizers require multi photolithography steps to form different layers, which makes the manufacturing of these devices a bit complicated.

One-dimensional Photonic crystals (1-D PCs) are the straightforward structures in a photonic crystal family, Intriguingly, these crystals still hold numerous stimulating characteristics such as modifiable dispersion and birefringence, analogous to homogeneous materials. The uncomplicated structure of the 1-D PC is easily integrated with the existing photonic devices without varying the fabrication process in comparison with 2-D or 3-D PCs. Accordingly, several high-performance silicon-based devices have been studied and demonstrated by making use of those exciting properties of 1-D PCs such as modulators [11], sensors [12], filters [13] and polarizer $[14,15]$, among others. In this letter, we proposed a compact design of a one-dimensional Photonic crystal strip waveguide (PCSW) capable of transmitting TM polarized light and rejecting the TE polarization. The waveguide design is based on a silicon-on-insulator (SOI) platform as shown in Fig. 1. The height and width of the silicon waveguide core are represented as $H$ and $W$, which is fixed at $300 \mathrm{~nm}$ and $450 \mathrm{~nm}$, respectively. A periodic array of rectangular cavity (void) is placed in the centre of the strip waveguide. A strip waveguide segment of $1 \mu \mathrm{m}$ is maintained on both ends of the device. The height of the rectangular cavity is equal to the height of the 
waveguide core. While the length and width of the cavity are denoted as $l$ and $W_{l}$, respectively. The gap between the two voids is denoted as $d$ while the period $(\Lambda)$ is $l+d$.

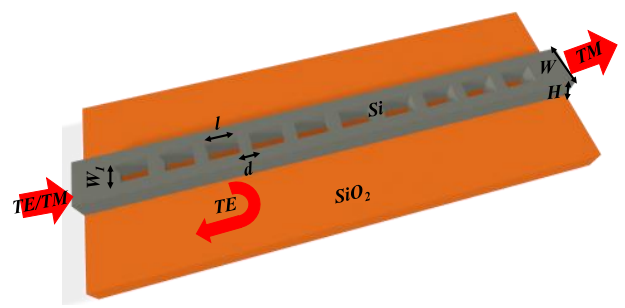

Fig. 1. Schematic representation of a proposed TM polarization pass 1Dphotonic crystal strip waveguide based on the SOI platform.

The device design is simulated using COMSOL Multiphysics 5.5 based 3D finite element method (FEM). The electromagnetic (EM) wave frequency domain (emw) is chosen as a physics interface. The waveguide model sub-domains are divided into triangular mesh elements with $\lambda / 20$ mesh grid size for the entire design. The meshing depends on the solution accuracy and the computational power of the system. For wave propagation systems, it is enviable to model a domain with open boundaries of the computational domain as it allows the electromagnetic wave to pass through without any reflection. The open geometry is set by assigning scattering boundary conditions (SBC) at the outer edges of the simulation window.
Here, we have optimized the PCSW structure by considering the geometric parameters of the PC. In the first step, the transmittance of the TE and TM mode at $1550 \mathrm{~nm}$ is calculated by varying the fill factor $(F F=l / 4)$ and $W_{l}$ of the PC. The number of period $(N)$ of the PC is initially maintained at 10 . The transmittance is calculated using the following expression:

Transmittance $(d B)=10 x \log \left(P_{\text {out }} / P_{\text {in }}\right)$, where $P_{\text {out }}$ and $P_{\text {in }}$ are the power at the output and input, respectively. For the optimization of 1D-PCSW, the $W_{l}$ and $F F$ are considered in the range of $30 \mathrm{~nm}$ to $400 \mathrm{~nm}$ and 0.25 to 0.75 , respectively. For $30 \mathrm{~nm}<W_{l}<175 \mathrm{~nm}$, TM polarized light has a transmission loss of $<1.9 \mathrm{~dB}$ for the $F F$ of $0.25-0.75$ and $W_{l}$ in the range of $30 \mathrm{~nm}$ to $175 \mathrm{~nm}$, whereas there is a bandgap for TE polarized light which shifts with varying $F F$. The maximum transmission loss of $26.14 \mathrm{~dB}$ is obtained at $W_{l}=175 \mathrm{~nm}$ and $F F=0.625$ as shown in Fig. 2a. In Figure 2b, the polarization extinction ratio $(P E R)$ is calculated for the transmittance of TE and TM polarized light obtained in Fig.2a. PER is the ratio of the output power of TM polarized light to the optical power of TE polarized light and is calculated using the following expression: PER $(d B)=10 x \log \left(\mathrm{P}_{\mathrm{TM}} / \mathrm{P}_{\mathrm{TE}}\right)$, where $\mathrm{P}_{\mathrm{TM}}$ and $\mathrm{P}_{\mathrm{TE}}$ are the optical power at $\mathrm{TM}$ and $\mathrm{TE}$ polarization, respectively.
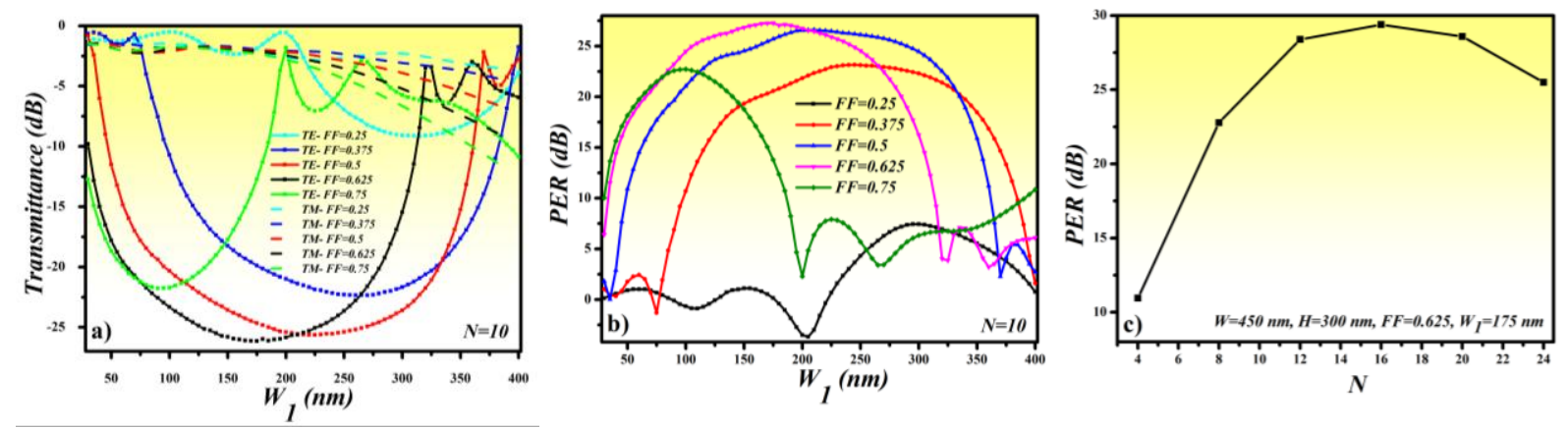

Fig. 2. a) Transmittance versus $W_{\text {hole }}$, b) $P E R$ versus $W_{\text {hole }}$, c) $P E R$ versus $N$.

In telecommunication, $P E R$ is used to illustrate the degree of polarization in polarization-maintaining devices or fiber. The maximum $P E R$ of $27.27 \mathrm{~dB}$ is obtained at $W_{l}=175 \mathrm{~nm}$ and $F F=0.625$ for $N=10$. Afterwards, the dependence of PER on $N$ is calculated for the optimized parameters of the PCSW. From Fig. 2c, it can be seen that as $N$ increases from 4 to 16 , the $P E R$ gradually increases from $10.9 \mathrm{~dB}$ to $29.4 \mathrm{~dB}$, which drops as $N$ continues to increase. Therefore, we have to find the optimum values of $W_{l}, F F$ and $N$ to have a maximum PER.

The normalized E-field distribution for TE and TM mode at $1550 \mathrm{~nm}$ is shown in Figs. $3 \mathrm{a}$ and $3 \mathrm{~b}$, respectively. The optimized geometric parameters of the PCSW is also shown in Fig 3. It is worth noting that TE polarized light suffers Bragg reflection at the designed waveguide parameters whereas TM mode travels without any loss. The E-field distribution of the TM mode at the crosssection of the waveguide is shown in Fig. 3c.

The transmittance of the proposed PCSW structure is directly related to $l$ of the PC as the bandgap can shift depending on the $\Lambda$. In Fig. 4, the transmittance of TE and TM polarized light is calculated versus $l$ while $d$ is kept constant at $150 \mathrm{~nm}$. $\Lambda$ is varied in the range of $250 \mathrm{~nm}$ to $550 \mathrm{~nm}$. 


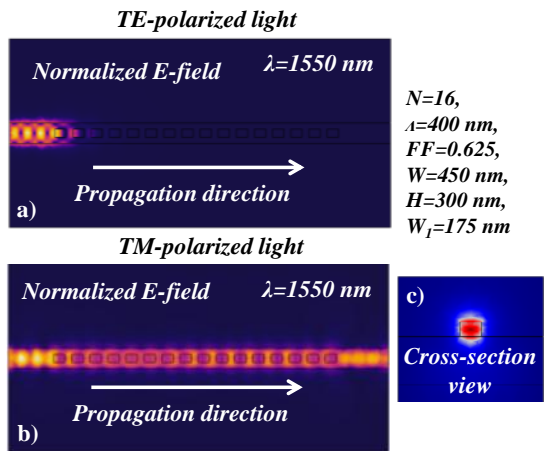

Fig. 3. Top view of a) normalized E-field distribution for TE-polarized light, b) normalized E-field distribution for TM polarized light, and c) cross-section view at TM polarized light.

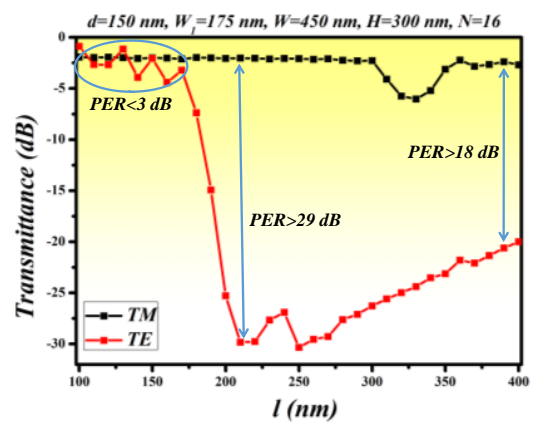

Fig. 4. Transmittance versus $l$, where $d$ is fixed at $150 \mathrm{~nm}$.

Here, we have used the optimized parameters obtained from Fig. 2, i.e. $W=450 \mathrm{~nm}, H=300 \mathrm{~nm}, W_{l}=175 \mathrm{~nm}$, $d=150 \mathrm{~nm}$ and $N=16$. From Fig. 4 , it can be seen that TM polarized light at $1550 \mathrm{~nm}$ has a constant transmittance of $\sim 2 \mathrm{~dB}$ for $l=100 \mathrm{~nm}$ to $300 \mathrm{~nm}$. From $l=300 \mathrm{~nm}$ to $l=350$ $\mathrm{nm}$, there is a fall in power to $6 \mathrm{Db}$, which recovers as the $l$ increases. However, for TE polarized light, the region where $200 \mathrm{~nm}<l<300 \mathrm{~nm}$ is interesting as PER is greater than $25 \mathrm{~dB}$.

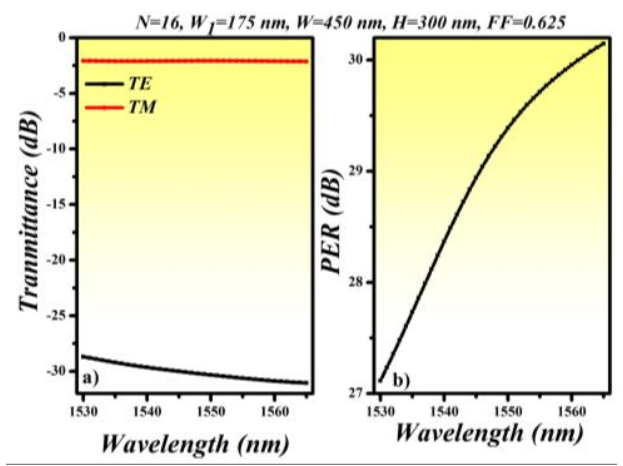

Fig. 5. a) Transmittance versus telecommunication C-band wavelength, b) PER versus telecommunication C-band wavelength.

In the end, we have simulated the optimized device design for the C-band telecommunication wavelengths to calculate the transmittance of TE and TM polarized light and PER as shown in Figs. 5 (a, b). In infrared optical communications, a C-band is dedicated to the wavelength range of $1530 \div 1565 \mathrm{~nm}$, which corresponds to the amplification range of an erbium-doped fiber amplifier. It can be seen that TM polarized light has a constant transmittance of $\sim 2 \mathrm{~dB}$ while it is in the range of $28 \mathrm{~dB}$ to $31 \mathrm{~dB}$ for TE polarized light for the whole C-band. Thus, it is concluded that our proposed device can work as a TM-polarized telecommunication C-band pass filter of length $8.4 \mu \mathrm{m}$, which offers a $P E R$ of $\sim 28.5 \mathrm{~dB}$.

In conclusion, the TM polarization C-band telecommunication wavelengths pass filter based on onedimensional photonic crystal strip waveguide is numerically studied. The waveguide structure is optimized to obtain the maximum transmittance of TM polarized light using the finite element method. The length, width and number of periods of the photonic crystal play an important role in the transmittance of the light (TE or TM). The optimized length of the device is approximately $8.4 \mu \mathrm{m}$, which includes $6.4 \mu \mathrm{m} \mathrm{PC}$ segment and $1 \mu \mathrm{m}$ of the strip waveguide segment on both ends of the device. The maximum transmittance of $\sim 2 \mathrm{~dB}$ of TM polarized light is obtained for the whole Cband telecommunication wavelengths for the following geometric parameters: $W=450 \mathrm{~nm}, H=300 \mathrm{~nm}, W_{l}=175$ $\mathrm{nm}, d=150 \mathrm{~nm}, l=250 \mathrm{~nm}, N=16$. However, for TE polarized light, the transmittance is at $\sim 28 \mathrm{~dB}$ to $30 \mathrm{~dB}$, which leads to a high PER of $27.1 \mathrm{~dB}$ to $30.1 \mathrm{~dB}$.

This work was financially supported by the Ministry of Science and Higher Education within the State assignment FSRC «Crystallography and Photonics» RAS (No. 007-GZ/Ch3363/26) for numerical calculations and Russian Science Foundation (No. 20-69-47110) for theoretical results.

\section{References}

[1] B. Wang, S. Blaize, R.S-Montiel, Nanoscale 11, 20685 (2019).

[2] D. Dai, J.E. Bowers, Nanophoton. 3, 283 (2014).

[3] M.A. Butt, S.N. Khonina, N.L. Kazanskiy, Comp. Opt. 43, 1079 (2019).

[4] M.A. Butt, S.N. Khonina, N.L. Kazanskiy, Laser Physics 28, 116202 (2018).

[5] M.A. Butt, S.N. Khonina, N.L. Kazanskiy, Laser Physics 29, 046207 (2019).

[6] Q. Wang, S.-T. Ho, IEEE Photonics J. 2, 49 (2010).

[7] C.-H. Chen, L. Pang, C.-H. Tsai, U. Levy, Y. Fainman, Opt. Expr. 13, 5347 (2005)

[8] S. Yuan, Y. Wang, Q. Huang, J. Xia, J. Yu, 11th International Conference on Group IV Photonics (GFP) (IEEE, 2014), pp. 183184.

[9] X. Guan, P. Chen, S. Chen, P. Xu, Y. Shi, D. Dai, Opt. Lett. 39, 4514 (2014).

[10] A.E.- S. Abd-Elkader, M.F.O. Hameed, N.F. Areed, H.E.-D. Mostafa, S.S. Obayya, J. Opt. Soc. Am. B. 36, 652 (2019).

[11] J. Li et al., J. Lightwave Technol. 31, 1601 (2013).

[12] N. Skivesen et al., Opt. Expr. 15, 3169 (2007).

[13] S. Lin, J. Hu, L. Kimerling, K. Crozier, Opt. Lett. 34, 3451 (2009).

[14] T. Liu, A.R. Zakharian, M. Fallahi, J.V. Moloney, M. Mansuripur, IEEE Photon. Technol. Lett. 17, 1435 (2005).

[15] R.K. Sinha, Y. Kalra, Opt. Expr. 14, 10790 (2006). 